PROCEEDINGS OF THE

AMERICAN MATHEMATICAL SOCIETY

Volume 127, Number 6, Pages 1801-1804

S 0002-9939(99)04720-6

Article electronically published on February 18, 1999

\title{
INVARIANT MANIFOLDS OF HYPERCYCLIC VECTORS FOR THE REAL SCALAR CASE
}

\author{
JUAN P. BÈS
}

(Communicated by Theodore W. Gamelin)

\begin{abstract}
We show that every hypercyclic operator on a real locally convex vector space admits a dense invariant linear manifold of hypercyclic vectors.
\end{abstract}

Given a locally convex vector space $X$ and a continuous operator $T: X \longrightarrow X$, we say that $\mathrm{T}$ is hypercyclic provided there exists some $x \in X$ whose orbit

$$
\operatorname{Orb}\{x, T\}=\left\{x, T x, T^{2} x, \ldots\right\}
$$

is dense in $X$. Such an $x$ is said to be a hypercyclic vector for $T$. A motivation for this definition comes from the invariant subset problem: $T$ has no non-trivial closed invariant subset if and only if every non-zero vector in $X$ is hypercyclic for $T$.

In 1990, B. Beauzamy constructed an example of an operator on a separable complex Hilbert space admitting a dense, invariant linear manifold whose non-zero vectors were all hypercyclic [3, Thm. A]. Using different techniques, G. Godefroy and J. Shapiro provided a large class of examples on Frèchet spaces of entire functions with the same property [5, Thm 5.1].

Soon after, D. Herrero [6, Proposition 4.1] and P. Bourdon [4] independently showed that every hypercyclic operator on a complex Hilbert space admits such a dense, invariant linear manifold of hypercyclic vectors (in fact, Bourdon's proof works for arbitrary complex locally convex spaces as well). We'd like to show here that the same holds for the real scalar case, by presenting a positive answer to the following question, raised by S. Ansari [1, Problem 1]:

"Suppose $X$ is a locally convex real vector space and $T: X \rightarrow X$ is a continuous linear operator with a hypercyclic vector $x$. Is it true that $P(T) x$ is a hypercyclic vector for $T$ whenever $\mathrm{P}$ is a non-zero polynomial with real coefficients?" (If so,

$$
\mathcal{M}=\{P(T) x: P \text { polynomial with real coefficients }\}
$$

is a dense, $T$-invariant manifold of hypercyclic vectors.)

Notice that since $P(T)$ and $T$ commute,

$$
\operatorname{Orb}\{P(T) x, T\}=P(T)(\operatorname{Orb}\{x, T\}) .
$$

That is, given $\mathrm{x}$ a hypercyclic vector for $T, P(T) x$ will also be hypercyclic if and only if $P(T)$ has dense range. So it will suffice for us to show the following.

Received by the editors September 17, 1997.

1991 Mathematics Subject Classification. Primary 47A15, 47A99.

The author wishes to thank the support of the Center for International and Comparative Programms and the Graduate Student Senate of Kent State University. 
Theorem. Let $X$ be a locally convex real vector space, and $T \in L(X)$ be hypercyclic. If $P$ is a non-zero polynomial with real coefficients, then $P(T)$ has dense range.

We'll make use of two results. The first one is due to C.Kitai [6, Theorem 2.3]:

Lemma 1. Let $X$ be a locally convex real (complex) vector space, and $T \in L(X)$ be hypercyclic. Then the adjoint $T^{*}$ of $T$ has no eigenvalues. In other words, for any scalar $b$, the operator $T+b I$ must have dense range.

Proof. Suppose $T^{*}$ admits an eigenvector $x$ with eigenvalue $\lambda$, and let $z \in X$ be a hypercyclic vector for $T$. Since $x \neq 0$, the set

$$
\left\{\left\langle x, T^{n} z\right\rangle\right\}_{n \geq 1}=\left\{\left\langle T^{* n} x, z\right\rangle\right\}_{n \geq 1}=\left\{\lambda^{n}\langle x, z\rangle\right\}_{n \geq 1}
$$

must be dense in the real (complex) scalar field, a contradiction.

The statement of the second result may be traced back at least to S. Rolewicz [8, p. 17]. We are grateful to P. Bourdon for suggesting the following argument to us.

Lemma 2. $R^{n}$ admits no hypercyclic operators.

Proof. Suppose there exists $A: R^{n} \rightarrow R^{n}$ linear with hypercyclic vector $z$. Notice that $B=\left\{z, A z, \ldots, A^{n-1} z\right\}$ must be linearly independent. Now, since $z$ is a hypercyclic vector for $A$, there exist sequences of positive integers $\left(n_{k}\right)$ and $\left(\tilde{n}_{k}\right)$ so that

$$
\begin{aligned}
& A^{n_{k}} z \underset{k \rightarrow \infty}{\rightarrow} 0, \\
& A^{\tilde{n}_{k}} z \underset{k \rightarrow \infty}{\longrightarrow} z .
\end{aligned}
$$

Because $B$ is a basis of $R^{n}$, we have in fact that

$$
\begin{aligned}
& A^{n_{k}} x \underset{k \rightarrow \infty}{\rightarrow} 0, \\
& A^{\tilde{n}_{k}} x \underset{k \rightarrow \infty}{\rightarrow} x
\end{aligned}
$$

for all $x \in R^{n}$. Hence, if $|A|$ denotes the determinant of $A$, the above lines imply the contradictory fact that

$$
\begin{aligned}
& |A|^{n_{k}}=\left|A^{n_{k}}\right| \underset{k \rightarrow \infty}{\rightarrow} 0, \\
& |A|^{\tilde{n}_{k}}=\left|A^{\tilde{n}_{k}}\right| \underset{k \rightarrow \infty}{\longrightarrow} 1 .
\end{aligned}
$$

So Lemma 2 holds.

Now, let's show the Theorem.

Proof of the Theorem. Since scalar multiples and compositions of operators having dense range have dense range, we may assume $P$ is irreducible and monic. Moreover, by Lemma 1 we may assume $P$ is of the form

$$
P(t)=t^{2}-2 R e(w) t+|w|^{2}, \quad \text { for some non-real complex number } w \text {. }
$$
Then

Now, suppose that $P(T)$ does not have dense range. Let $0 \neq x \in \operatorname{Ker}\left(P(T)^{*}\right)$.

$$
T^{* 2} x=a_{2} T^{*} x+b_{2} x, \text { where }\left\{\begin{array}{lll}
a_{2} & = & w+\bar{w} \\
b_{2} & = & -|w|^{2}
\end{array}\right.
$$


By Lemma $1,\left\{T^{*} x, x\right\} \subset \operatorname{Ker}\left(P(T)^{*}\right)$ must be linearly independent. So there exist unique scalars $a_{n}$ and $b_{n}$ satisfying

$$
T^{* n} x=a_{n} T^{*} x+b_{n} x \quad(n=1,2, \ldots) .
$$

Notice that by $(2)$

$$
\begin{aligned}
T^{* n+1} x & =T^{*}\left(a_{n} T^{*} x+b_{n} x\right) \\
& =a_{n}\left(a_{2} T^{*} x+b_{2} x\right)+b_{n} T^{*} x \\
& =\left(a_{n} a_{2}+b_{n}\right) T^{*} x+a_{n} b_{2} x .
\end{aligned}
$$

That is,

$$
\left[\begin{array}{l}
a_{n+1} \\
b_{n+1}
\end{array}\right]=A\left[\begin{array}{l}
a_{n} \\
b_{n}
\end{array}\right], \quad \text { where } A=\left[\begin{array}{ll}
a_{2} & 1 \\
b_{2} & 0
\end{array}\right]=\left[\begin{array}{cc}
w+\bar{w} & 1 \\
-|w|^{2} & 0
\end{array}\right] .
$$

So for all $n \geq 2$,

$$
\left[\begin{array}{l}
a_{n} \\
b_{n}
\end{array}\right]=A^{n-2}\left[\begin{array}{l}
a_{2} \\
b_{2}
\end{array}\right]
$$

and

$$
\left[\begin{array}{ll}
a_{2} & b_{2} \\
a_{3} & b_{3}
\end{array}\right]\left(A^{n-2}\right)^{t}=\left[\begin{array}{cc}
a_{n} & b_{n} \\
a_{n+1} & b_{n+1}
\end{array}\right] .
$$

Next, notice that since $\left\{T^{*} x, x\right\}$ is linearly independent, the operator

$$
\begin{aligned}
& X \stackrel{\Pi}{\stackrel{\Pi}{\longrightarrow}} R^{2} \\
& y \mapsto\left(\langle x, y\rangle ;\left\langle T^{*} x, y\right\rangle\right)
\end{aligned}
$$

is onto. In particular, if $z \in X$ is a hypercyclic vector for $T$, then

$$
\left\{\Pi\left(T^{n} z\right)\right\}_{n \geq 1}
$$

must be dense in $R^{2}$.

Hence, by (2) and (4) we have for each $n \geq 2$

$$
\begin{aligned}
\Pi\left(T^{n} z\right) & =\left(\left\langle x, T^{n} z\right\rangle ;\left\langle T^{*} x, T^{n} z\right\rangle\right) \\
& =\left(\left\langle T^{* n} x, z\right\rangle ;\left\langle T^{* n+1} x, z\right\rangle\right) \\
& =\left(\left\langle a_{n} T^{*} x+b_{n} x, z\right\rangle ;\left\langle a_{n+1} T^{*} x+b_{n+1} x, z\right\rangle\right) \\
& =\left[\begin{array}{cc}
a_{n} & b_{n} \\
a_{n+1} & b_{n+1}
\end{array}\right]\left[\begin{array}{c}
\left\langle T^{*} x, z\right\rangle \\
\langle x, z\rangle
\end{array}\right] \\
& =\left[\begin{array}{cc}
a_{2} & b_{2} \\
a_{3} & b_{3}
\end{array}\right]\left(A^{n-2}\right)^{t}\left[\begin{array}{c}
\left\langle T^{*} x, z\right\rangle \\
\langle x, z\rangle
\end{array}\right] .
\end{aligned}
$$

Thus, (5) and (6) force $A^{t}$ to be hypercyclic on $R^{2}$, which contradicts Lemma 2. So $P(T)$ must have dense range.

Remark. As a consequence of the theorem, S. Ansari's proof that every operator on a complex Banach Space shares with its powers the same hypercyclic vectors $[2$, Thm. 1] works for the real scalar case as well (see also [1, Note 3$]$ ). 


\section{REFERENCES}

1. S. I. Ansari, Existence of Hypercyclic Operators on Topological Vector Spaces, J. Funct. Anal. 148 (1997), no. 2, 384-390. CMP 98:01

2. S. I. Ansari, Hypercyclic and Cyclic Vectors, J. Funct. Anal. 128 (2) (1995). MR 96h:47002

3. B. Beauzamy, An operator on a separable Hilbert space with all polynomials hypercyclic, Studia Math. T. XCVI (1990), 81-90. MR 91d:47004

4. P. Bourdon, Invariant Manifolds of Hypercyclic Vectors, Proc. Amer. Math. Soc. 118 (3) (1993), 845-847. MR 93i:47002

5. G. Godefroy and Joel H. Shapiro, Operators with Dense, Invariant, Cyclic Vector Manifolds, J. Funct. Anal. 98 (1991), 229-269. MR 92d:47029

6. Domingo A.Herrero, Limits of Hypercyclic and Supercyclic Operators, J. Funct. Anal. 99 (1991), 179-190. MR 92g:47026

7. C. Kitai, Invariant Closed Sets for Linear Operators, Thesis, University of Toronto (1982).

8. S. Rolewicz, On orbits of elements, Studia Math. T. XXXII (1969), 17-22. MR 39:3292

Department of Mathematics and Computer Science, Kent State University, Kent, Оніо 44242

E-mail address: jbes@mcs.kent.edu

Current address: Department of Mathematics and Statistics, Bowling Green State University, Bowling Green, Ohio 43403

E-mail address: jbes@math.bgsu.edu 